Case Report

\title{
Fungal nasopharyngitis masquerading as nasopharyngeal mass in an immunocompetent patient: a case report
}

\section{P. Karthikeyan, Nikhil Sivanand*, Neelima Vijayan, Vishnu Kumar, Nanditha Srinivasa, Vignesh Raghavan}

\begin{abstract}
Department of Otorhinolaryngology and Head and Neck Surgery, Mahatma Gandhi Medical College and Research Institute Pondicherry, India
\end{abstract}

Received: 04 June 2019

Revised: 04 July 2019

Accepted: 11 July 2019

\section{*Correspondence:}

Dr. Nikhil Sivanand,

E-mail: nikhil.sivanand@gmail.com

Copyright: (C) the author(s), publisher and licensee Medip Academy. This is an open-access article distributed under the terms of the Creative Commons Attribution Non-Commercial License, which permits unrestricted non-commercial use, distribution, and reproduction in any medium, provided the original work is properly cited.

\begin{abstract}
Isolated nasopharyngeal candidiasis in an immunocompetent host, has not been reported in literature. This is a case of a 48 year old lady with no comorbidities who presented with a mass in the nasopharynx mimicking nasopharyngeal malignancy. Endoscopic debridement and biopsy revealed necrotic tissue with fungal ball composed of filamentous hyphae suggestive of aspergillus and budding yeast form of Candida. A diagnosis of fungal nasopharyngitis was made and started on antifungal therapy for 6 weeks following which patient is relieved of symptoms.
\end{abstract}

Keywords: Nasopharyngeal, Candidiasis, Endoscopic debridement, Fluconazole

\section{INTRODUCTION}

Clinical symptoms and imaging features of fungal infection are confused with those of atypical nasopharyngeal carcinoma (NPC). The development of an effective diagnostic method is therefore essential. The association between Epstein-Barr virus (EBV) and non keratinizing NPC is well known. ${ }^{1}$ However, fungal infection may be considered to be a differential diagnosis in a suspected case of NPC and proper investigation and timely intervention can help in better treatment outcome for the patient.

Candidiasis or oral thrush is a fungal infection caused by any of the Candida species, of which Candida albicans is the most common etiological agent. Candidiasis is often known as the "disease of the diseased" as it is seen in cases when the immunity of the host is suppressed or compromised. Nasopharyngeal or oropharyngeal candidiasis is one of the complications that may be associated with long-term use of steroid inhalers. Whenever an asthmatic patient who is on steroid inhalers reports with oral mucosal lesion, steroid-induced oropharyngeal candidiasis must be suspected. ${ }^{2}$ Isolated nasopharyngeal candidiasis in an immunocompetent host has not been reported in literature.

We report a case of fungal nasopharyngitis who presented to our department with atypical nasal complaints and timely debridement and antifungal therapy helped in saving the life of the patient.

\section{CASE REPORT}

48 year old home maker hailing from Pondicherry presented to the Emergency Department with chief complaints of dental pain, difficulty in swallowing and nasal obstruction since the last 5 days. Her past medical and surgical history was unremarkable.

On examination, she had muffled voice with toxic look and was febrile. Oral cavity examination revealed poor oral hygiene with halitosis and dental caries in bilateral lower premolars. Oropharyngeal examination showed oedematous and congested soft palate with diffuse swelling and uvula was oedematous and congested. 
Slough was present in the posterior pharyngeal wall with post nasal drip. Neck examination revealed a diffuse swelling in the submandibular triangle with tenderness and tender lymph nodes in the level II and level III on both sides. Both the tympanic membranes were retracted with grade 2 retraction of pars tensa.

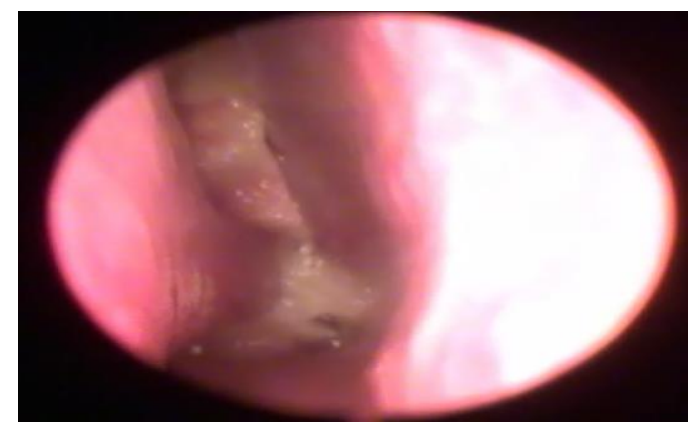

Figure 1: Preoperative nasal endoscopy showing slough covered mass arising from the nasopharynx extending anteriorly into the nasal cavity.

On nasal endoscopy, a slough covered mass was seen filling the choana on both sides with thick mucopurulent discharge in both the nasal cavities (Figure 1). Laryngoscopy showed slough in the posterior pharyngeal wall extending till the level of the epiglottis. Bilateral vocal cords were mobile and pyriform fossae free (Figure 2).

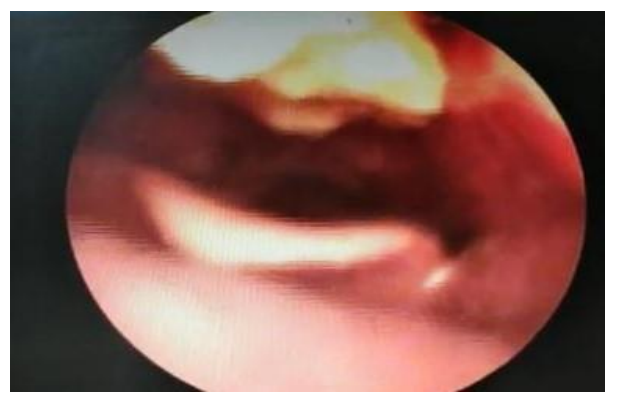

Figure 2: Preoperative videolaryngoscopy showing slough covered mass in the posterior pharyngeal wall.

\section{Diagnostic assessment}

An initial diagnosis of acute nasopharyngitis and early Ludwig's angina were considered and routine investigations done. She was started empirically on Injectible broad spectrum antibiotics (Piperacilin Tazobactem $4.5 \mathrm{~g}$ TID, Metronidazole $500 \mathrm{mg}$ TID) and one dose of hydrocortisone was given in view of oropharyngeal oedema.

Routine blood workup showed elevated total counts $(21,400$ cells $/ \mathrm{cmm})$ with mild neutrophilia and platelet counts were $1,50,000 / \mathrm{cu} . \mathrm{mm}$. ESR was elevated to 40 $\mathrm{mm} / \mathrm{hr}$. RFT and sugars were normal and Serology was negative. Throat swab was sent for culture and after 48 hours it showed fungal elements (non Albicans candida).
Ultrasound neck revealed oedematous right submandibular gland, bilateral cervical lymphadenitis (necrotic lymph nodes) with inflammatory changes adjacent to it. No obvious evidence of collection/abscess noted in the floor of mouth or in the submandibular region.

X-ray of soft neck lateral view neck was done to look for any airway compromise of the airway, which was patent, but showed soft tissue density in the nasopharyngeal region (Figure 3 ).

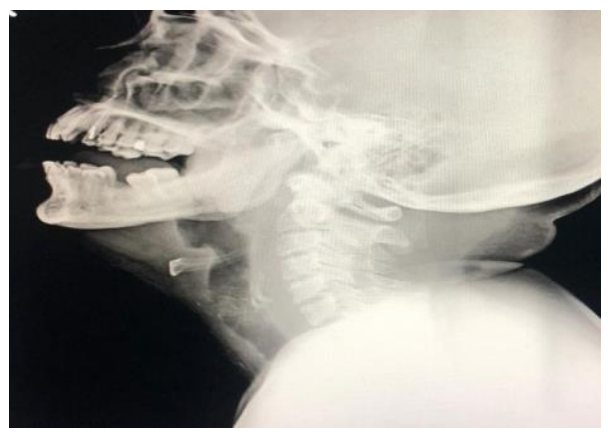

Figure 3: X-ray neck lateral view showing soft tissue opacity in the nasopharynx pushing the soft palate inferiorly.

CT scan of nose and paranasal Sinuses revealed an illdefined soft tissue density lesion involving the nasopharyngeal and the oropharyngeal lumen with a few air pockets/ bubbly secretions, with complete obliteration of the bilateral posterior choana- the tonsillar fossae were obliterated and the soft palate was not clearly visualized (Figure 4).

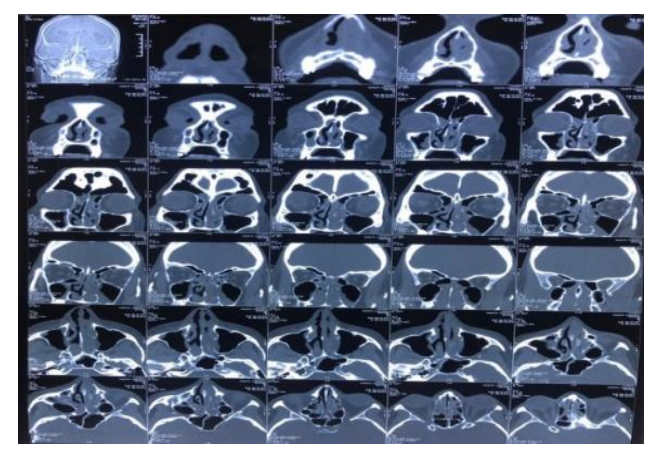

Figure 4: CT nose and PNS showing soft tissue opacity in bilateral choana completely obstructing and extending towards the nasal cavity and oropharynx.

After routine investigations, the working diagnosis was changed to nasopharyngeal carcinoma with secondaries in the neck and tuberculosis of the nasopharynx. Pulmonologists opinion was taken and Monteux test came as negative. Patient underwent endoscopic debridement of nasopharyngeal mass and biopsy under GA and the nasopharyngeal mass was removed in toto and sent for histopathology. Postoperatively her symptoms improved drastically and the nasal pack was 
removed on the second day following which she received nasal douching she was continued on Intravenous antibiotics for 5 days.

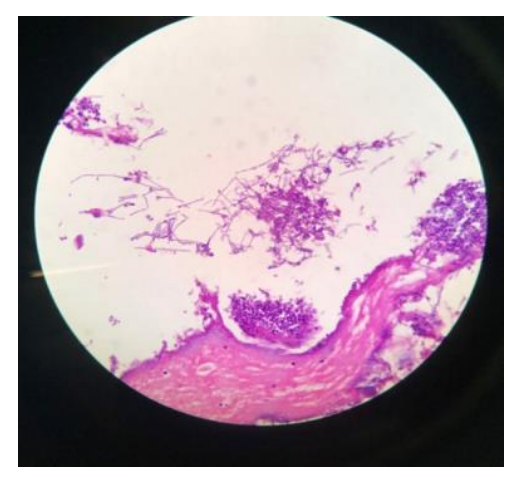

Figure 5: H\&E stained image hemorrhagic and necrotic tissue with a fungal ball composed of filamentous fungus and inflammatory cells (10X).

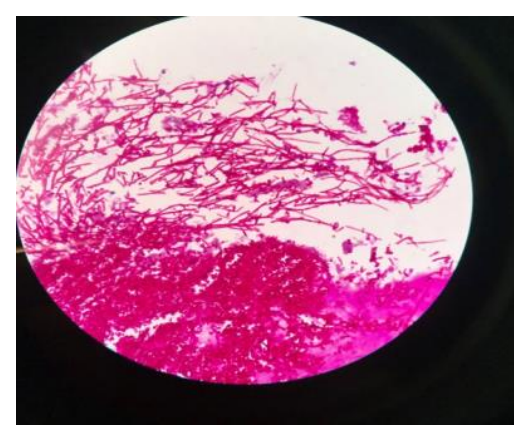

Figure 6: Histopathology showing PAS positive hyphal forms of filamentous fungus (40X).

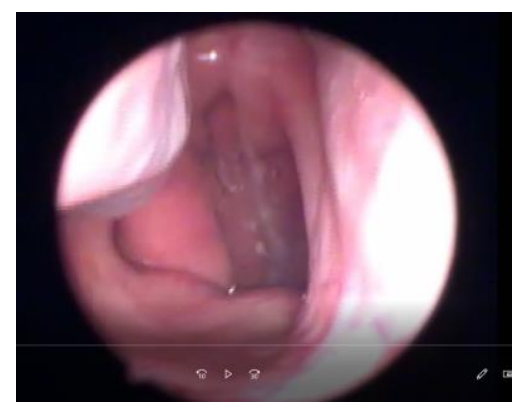

Figure 7: Follow-up (4th week postoperative) showing complete clearance of disease from the choana on nasal endoscopy.

Histopathology report reported as hemorrhagic and necrotic tissue with a fungal ball composed of filamentous fungus suggestive of aspergillus with budding form of Candida with pseudohyphae. No evidence of carcinoma seen in the sections studied (Figure 5 and 6).

After histopathology report, patient was started on oral fluconazole $200 \mathrm{mg}$ stat followed by oral fluconazole 100 mg once a week for 6 weeks. Patient is free of symptoms and on routine follow-up (Figure 7).

\section{DISCUSSION}

The incidence of fungal infection is increasing in the recent times which are mainly due to the use of high dose broad-spectrum antibiotics over a long period, high dose steroids/cytotoxic chemotherapy, immunosuppressant drugs and chemotherapeutics. The main pathogens are still Candida albicans and non-albicans Candida species. $^{3}$ The association between the incidence of fungal infections in the aero digestive tract and the use of inhalations steroids are well understood. ${ }^{4}$ Isolated fungal nasopharyngitis in an immunocompetent host has not been described in literature before. It is a diagnostic challenge as it presents with atypical symptoms like nasal block, nasal discharge and rhinolalia clausa. Fungal nasopharyngitis needs debridement of the mass if present with systemic administration of antifungal medications. Recognizing that this process is primarily fungal in origin is paramount to successful treatment, as most patients respond well to antifungal therapy when compliant with treatment. Bacterial and viral nasopharyngitis are a common entity. However, fungal nasopharyngitis has not been reported in literature and it requires systemic antifungal treatment for upto 6 weeks which is not required for both bacterial and viral nasopharyngitis.

This particular case presented with complaints of a nasopharyngeal block and acute nasopharyngitis was considered. Since she is an immunocompetent patient, the probability of it being a fungal condition was less likely. However, timely debridement and evaluation proved a fungal aetiology and patient has been relieved from her symptoms completely with postoperative antifungal therapy. This case report throws light on the incidence of fungal nasopharyngitis in an immunocompetent host.

\section{CONCLUSION}

We present a rare case of fungal nasopharyngitis in an immunocompetent host which has not been reported before. This finding is especially important because timely debridement and antifungal therapy can completely relieve the patient of their symptoms and improve the quality of life. It is necessary to seek timely, effective management to decrease both the tissue burden and the risk of toxaemia and sepsis. It is therefore important to suspect fungal nasopharyngitis even in an immunocompetent host.

\section{ACKNOWLEDGEMENTS}

The authors would like to acknowledge the patient, relatives, nursing staff and pathology department for providing required help.

\section{Funding: No funding sources \\ Conflict of interest: None declared \\ Ethical approval: Not required}




\section{REFERENCES}

1. Ren K, Wang W, Ma X, Guo F, Li P, Liu L. Nasopharyngeal carcinoma mimicking Aspergillosis rhinosinusitis: an unusual case report and review of the literature. Int $\mathbf{J}$ Clin Exp Pathol. 2014;7(12):9050-5.

2. John P, Jayasree VM. Oropharyngeal candidiasis associated with use of steroid inhaler in a chronic asthmatic patient: case report. Int J Oral Maxillofac Pathol. 2013:4(3):40-4.
3. Zhengyin L, Liu ZY, Sheng RY, Li XL , Li TS, Wang AX. Analysis of 149 cases of fungal infection in hospitals. Chinese J Med. 2003;83(05):399-402.

4. Buhl R. Local oropharyngeal side effects of inhaled corticosteroids in patients with asthma. Allergy. 2006;61:518-26.

Cite this article as: Karthikeyan P, Sivanand N, Vijayan N, Kumar V, Srinivasa N, Raghavan V. Fungal nasopharyngitis masquerading as nasopharyngeal mass in an immunocompetent patient: a case report. Int J Otorhinolaryngol Head Neck Surg 2019;5:1407-10. 